Wright State University

CORE Scholar

$1-1-1997$

\title{
Identification of Electron-Irradiation Defects in Semi-Insulating GaAs by Normalized Thermally Stimulated Current Measurements
}

\author{
David C. Look \\ Wright State University - Main Campus, david.look@wright.edu \\ Z-Q. Fang \\ Joseph W. Hemsky \\ Wright State University - Main Campus, joseph.hemsky@wright.edu \\ P. Kengkan
}

Follow this and additional works at: https://corescholar.libraries.wright.edu/physics

Part of the Physics Commons

\section{Repository Citation}

Look, D. C., Fang, Z., Hemsky, J. W., \& Kengkan, P. (1997). Identification of Electron-Irradiation Defects in Semi-Insulating GaAs by Normalized Thermally Stimulated Current Measurements. Physical Review B, 55 (4), 2214-2218.

https://corescholar.libraries.wright.edu/physics/201

This Article is brought to you for free and open access by the Physics at CORE Scholar. It has been accepted for inclusion in Physics Faculty Publications by an authorized administrator of CORE Scholar. For more information, please contact library-corescholar@wright.edu. 


\title{
Identification of electron-irradiation defects in semi-insulating GaAs by normalized thermally stimulated current measurements
}

\author{
D. C. Look, Z-Q. Fang, J. W. Hemsky, and P. Kengkan* \\ Physics Department, Wright State University, Dayton, Ohio 45435
}

(Received 19 September 1996)

\begin{abstract}
Primary defects induced by $1 \mathrm{MeV}$ electron irradiation have been quantitatively studied in semi-insulating (SI) GaAs by using normalized thermally stimulated current spectroscopy, a new technique. Defects identical to (or similar to) those known in the thermally stimulated current literature as $T_{6}^{*}(0.13 \mathrm{eV}), T_{5}(0.34 \mathrm{eV})$, and $T_{4}(0.31 \mathrm{eV})$ are produced at rates $0.70,0.08$, and $0.23 \mathrm{~cm}^{-1}$, respectively; $T_{5}$ is also a strong trap in unirradiated SI GaAs. The defects $T_{6}^{*}$ and $T_{4}$ correspond closely to the irradiation-induced traps $E 2(0.14 \mathrm{eV})$ and E3 $(0.30 \mathrm{eV})$, studied extensively by deep-level transient spectroscopy and Hall-effect measurements and assigned to the As vacancy. We thus infer that traps $T_{6}^{*}$ and $T_{4}$ (and probably also $T_{5}$ ) in SI GaAs have As-vacancy character. [S0163-1829(97)04503-7]
\end{abstract}

\section{INTRODUCTION}

High-energy electron irradiation has been employed to study primary defects (vacancies, interstitials, and, sometimes, antisites) in many metal and semiconductor materials. ${ }^{1}$ Typically, the electron energy necessary to displace an atom will be a few hundred $\mathrm{keV}$; thus, the common choice of $1-\mathrm{MeV}$ irradiation will produce only one, or at most a few, displacements and no massive damage, such as is often found with heavy-ion irradiation. Semiconducting GaAs has been investigated in this manner for several decades (for reviews, see Refs. 2 and 3). Many techniques have been employed, but quantitative analysis has mainly relied on temperature-dependent Hall-effect (TDH) (Ref. 4) and deeplevel transient spectroscopy (DLTS) measurements, or other methods involving capacitance. ${ }^{3}$ However, the TDH and DLTS techniques cannot be applied in semi-insulating (SI) GaAs, an important material that forms the basis of the GaAs microwave and integrated-circuit industries. A wellestablished method for looking at traps in SI materials is thermally stimulated current (TSC) spectroscopy; ${ }^{5-8}$ however, TSC is not considered to be a quantitative technique because it involves carrier mobility, lifetime, and geometric factors, which are either unknown or poorly known. In this work we first show how to quantify a TSC spectrum, by normalizing with infrared photocurrent, and then apply this quantitative method (called NTSC) to study traps produced by electron irradiation in SI GaAs. The NTSC traps $T_{6}^{*}(0.13 \mathrm{eV})$ and $T_{4}(0.31 \mathrm{eV})$, which sometimes appear in as-grown (unirradiated) SI GaAs, are shown to be equivalent to the DLTS electron traps $E 2(0.14 \mathrm{eV})$ and $E 3(0.30 \mathrm{eV})$, respectively, and are assigned to the As vacancy $V_{\mathrm{As}}$ or its complex. Another defect, $T_{5}(0.34 \mathrm{eV})$, which is always prominent in as-grown SI GaAs, grows with irradiation, but at a smaller rate than that found for either $T_{6}^{*}$ or $T_{4}$. The other two most prominent TSC traps in as-grown SI GaAs, $T_{2}(0.63 \mathrm{eV})$ and $T_{3}(0.50 \mathrm{eV})$, are unaffected by $1-\mathrm{MeV}$ irradiation.

\section{THEORY}

The idea behind the normalization procedure is that both TSC and infrared (IR) photocurrent (PC) are linearly propor- tional to carrier lifetime $\tau(T)$, mobility $\mu(T)$, and certain geometric factors; ${ }^{5}$ thus, their ratio $\left(I_{\mathrm{TSC}} / I_{\mathrm{PC}}\right)$ mostly involves only quantities that are either known or can be fitted. The only remaining unknown quantity, in general, is the absorption coefficient $\alpha(T)$; however, fortunately, for IR excitation of electrons from EL2 (the $\mathrm{As}_{\mathrm{Ga}}$-related defect that is dominant in SI GaAs), $\alpha(T)$ is well known. ${ }^{9,10}$ The TSC for electron traps can be shown to obey ${ }^{5}$

$$
I_{\mathrm{TSC}}=e \mu_{n} \tau_{n} \frac{w d}{l} V N_{T} e_{n} \exp \left(-\int_{T_{0}}^{T} \frac{e_{n}}{\beta} d T^{\prime}\right),
$$

where a rectangular sample is assumed (length $\ell$, width $w$, and thickness $d), V$ is the applied voltage, $N_{T}$ the trap density, $T_{0}$ the starting temperature, $\beta$ the heating rate, and $e_{n}$ the emission rate given by

$$
e_{n}=\frac{16 \pi \mathrm{m}_{n}^{*} k^{2}}{h^{3}}\left(\frac{g_{0}}{g_{1}} \sigma_{n 0} e^{\alpha_{T} / k}\right) T^{2} e^{-\left(E_{0}+E_{\sigma}\right) / k T} .
$$

Here, $g_{0}\left(g_{1}\right)$ is the degeneracy of the unoccupied (occupied) state, $\sigma_{n}=\sigma_{n 0} \exp \left(-E_{\sigma} / k T\right)$ is the electron capture cross section, $\alpha_{T}$ is defined by $E=E_{0}-\alpha_{T} T$, and $E$ is the energy of the trap defined with respect to the conduction band. Often the term $\left(g_{0} / g_{1}\right) \sigma_{n 0} \exp \left(\alpha_{T} / k\right)$ is called the apparent capture cross section $\sigma_{a}$, and $\left(E_{0}+E_{\sigma}\right)$, the apparent energy, $E_{a}$. For a nonrectangular sample, the factor $w d / \ell$ will change; however, it will cancel out anyway in the ratio $I_{\mathrm{TSC}} / I_{\mathrm{PC}}$ as shown below.

We now turn to the derivation of the PC under illumination by IR light $\left(1.1 \mu \mathrm{m}\right.$ in our case) of intensity $I_{0}$ photons $/ \mathrm{cm}^{2}$ s. In a thick sample $(\alpha d \gg 1$, where $\alpha$ is the absorption coefficient), all of the light will be absorbed except for that reflected by the front surface; in other words, the effective light intensity is $I_{0}(1-R)$. However, in a thin sample, multiple reflections involving the back surface also must be considered. Consider a small region at distance $x$ from the sample surface and of length $d x$; then the volume 
concentration of electrons $n_{1 d}$ produced on the first downward pass of the light (before reflection at the back surface) is

$$
\begin{aligned}
n_{1 d} & =\frac{1}{d} \int_{0}^{d} I_{0}(1-R) \alpha_{n} e^{-\alpha x} \tau_{n} d x \\
& =I_{0}(1-R) \alpha_{n} \tau_{n} \frac{1-e^{-\alpha d}}{\alpha d},
\end{aligned}
$$

where $\alpha_{n}$ is the portion of the absorption which produces free electrons and $\alpha$ is the total absorption. The intensity of light reaching the backside is $I_{0}(1-R) \exp (-\alpha d)$ and the intensity reflected back toward the upper surface is $I_{0} R(1-R) \exp (-\alpha d)$. Thus, the concentration of electrons produced during the first upward pass is $n_{1 u}$ $=R \exp (-\alpha d) n_{1 d}$. On the second downward pass the concentration is $n_{2 d}=R^{2} \exp (-2 \alpha d) n_{1 d}$, and the final result, after many passes, is

$$
n=n_{1 d}+n_{1 u}+n_{2 d}+\cdots=I_{0} \alpha_{n} \tau \frac{1-e^{-\alpha d}}{\alpha d} \frac{1-R}{1-\mathrm{Re}^{-\alpha d}} .
$$

For SI GaAs, $R(1.1 \mu \mathrm{m}) \simeq 0.305$, and typically $\alpha \simeq \alpha_{n} \simeq 1$ $\mathrm{cm}^{-1}$ and $d \simeq 0.06 \mathrm{~cm}$. Thus, $\alpha d \ll 1$, and $n \simeq I_{0} \alpha_{n} \tau$. This derivation reveals an interesting fact: for a very thin sample, the effectively longer path length due to the multiple reflections in the sample itself exactly makes up for the reflection lost from the front surface.

The $\mathrm{PC}$ is then given by $I_{\mathrm{PC}}=e n \mu_{n}(w d / \ell) V$ so that $I_{\mathrm{TSC}} / I_{\mathrm{PC}}$ becomes

$$
\frac{I_{\mathrm{TSC}}}{I_{\mathrm{PC}}}=\frac{N_{T} e_{n} \exp \left(-\int_{T_{0}}^{T} \frac{e_{n}}{\beta} d T^{\prime}\right)}{I_{0} \alpha_{n} \frac{1-e^{-\alpha d}}{\alpha d} \frac{1-R}{1-\mathrm{Re}^{-\alpha d}}} .
$$

Here we are neglecting the PC due to holes since $\alpha_{p}(1.1$ $\mu \mathrm{m})<\alpha_{n}(1.1 \mu \mathrm{m})$ and $\mu_{p} \ll \mu_{n}$. For SI GaAs, $\alpha_{n}(\lambda)$ $=\sigma_{\nu n}(\lambda) N_{E L 2}^{0}$ and $\alpha(\lambda)=\sigma_{\nu n}(\lambda) N_{E L 2}^{0}+\sigma_{\nu p}(\lambda) N_{E L 2}^{+}$. The photoionization coefficients, $\sigma_{\nu n}$ and $\sigma_{\nu p}$, for $\lambda=1.1 \mu \mathrm{m}$, are well known. ${ }^{9,10}$ Also, $N_{E L 2}^{0}$ and $N_{E L 2}^{+}$can be determined from transmission measurements at two different wavelengths, say 1.1 and $1.2 \mu \mathrm{m} .{ }^{10}$ Thus, a fit of $I_{\mathrm{TSC}} / I_{\mathrm{PC}}$ as a function of sweep temperature, $T=T_{0}+\beta t$, will give $N_{T}$, $\sigma_{a}$, and $E_{a}$ as fitting parameters. However, in general, $\sigma_{a}$ cannot be fitted accurately, as is also the case in DLTS analysis (e.g., see Ref. 11, p. 202).

Equation (5) assumes that all of the $N_{T}$ traps are filled by the illumination, which will not be true if the illumination excites electrons out of the trap even while it is providing conduction-band electrons from other sources (EL2 or the valence band) which can be captured by the trap. In steady state (long illumination time) the occupied fraction will be

$$
\frac{N_{T}^{0}}{N_{T}}=\frac{n v_{n} \sigma_{n}+I_{0} \sigma_{\nu p T}(\lambda)}{n v_{n} \sigma_{n}+p v_{p} \sigma_{p}+I_{0}\left[\sigma_{\nu n T}(\lambda)+\sigma_{\nu p T}(\lambda)\right]},
$$

where $\sigma_{\nu n T}(\lambda)$ is the photoionization cross section for electron excitation from the trap $T$ to the conduction band by light of wavelength $\lambda$, and $\sigma_{\nu p T}(\lambda)$ is the analogous term for hole excitation. For trap filling, we used $1.45-\mathrm{eV}$ light, an energy just below the band gap at $83 \mathrm{~K}$, the temperature at which the traps were filled. The reason for filling with $1.45-\mathrm{eV}$ light, rather than the $1.1-\mu \mathrm{m}$ light used for the PC, is that the latter can also cause $E L 2$ quenching, which complicates the analysis. ${ }^{7}$ To test the importance of Eq. (6) in the present analysis, we employed a wide range of filling wavelengths $\lambda$ and intensities $I_{0}$, and measured $N_{T}$ (or really $N_{T}^{0}$ ) for each. The conclusion was that only trap $T_{5}$ was affected much by $\lambda$ or $I_{0}$, but that even for $T_{5}$, the chosen conditions $\left[\lambda=0.855 \mu \mathrm{m}(1.45 \mathrm{eV})\right.$ and $I_{0}=3.3 \times 10^{14}$ photons $\left./ \mathrm{cm}^{2} \mathrm{~s}\right]$ gave nearly the maximum peak height. Thus, we believe that the ratio $N_{T}^{0} / N_{T}$ in our experiment is within $10 \%$ of unity for all of the traps, $T_{6}^{*}, T_{5}$, and $T_{4}$.

For trap $T_{6}^{*}$, which has a peak at $91 \mathrm{~K}$, we must also consider emission during the 30-s time interval that the sample sits at $83 \mathrm{~K}$ after the light has been turned off but before the sweep has begun. Fortunately, the fitting of Eq. (5) to the NTSC spectrum gives $e_{n}(T)$ [Eq. (2)] and therefore the loss of neutral (filled) $T_{6}^{*}$ traps can be easily calculated:

$$
\frac{\left[T_{6}^{*}\right]^{0}(t)}{\left[T_{6}^{*}\right]^{0}(0)}=e^{-e_{n}(83 \mathrm{~K}) t}
$$

where $t=30 \mathrm{~s}$ in our case. It turns out that the loss is about $25 \%$, and this factor must be included in the analysis.

Finally, we must analyze the effects of electron energy loss in a thick sample. Let $E_{d}$ be the absorbed energy necessary to displace an atom $\left(E_{d} \simeq 10 \mathrm{eV}\right.$ in GaAs). ${ }^{3}$ Then the minimum (threshold) electron energy $E_{t}$ that will transfer at least $E_{d}$ to an atom of mass $M$ is given by (Eq. 1.46, Ref. 1).

$$
E_{d}=2\left(\frac{m_{e}}{M}\right) \frac{E_{t}\left(E_{t}+2 m_{e} c^{2}\right)}{m_{e} c^{2}} .
$$

Thus, $E_{t} \simeq 0.27 \mathrm{MeV}$ for $E_{d}=10 \mathrm{eV}$. A numerical calculation using electron energy-loss theory ${ }^{12}$ (most of the loss is due to electronic collisions) gives a range of $970 \mu \mathrm{m}$ for an initially $1-\mathrm{MeV}$ electron to fall to $0.27 \mathrm{MeV}$. Thus, appreciable energy loss will occur in a typical 600-700- $\mu$ m-thick sample, and more displacements will occur near the upper surface than the lower surface. This situation is discussed in Ref. 1, and Eq. 1.57 of that reference can be rewritten as follows:

$$
N_{T}=N_{T}(E)\left\{1-\frac{d}{2\left[R(E)-R\left(E_{\mathrm{th}}\right)\right]}\right\},
$$

where $E=1 \mathrm{MeV}$, and $E_{\text {th }}=0.27 \mathrm{MeV}$, in this case. Thus, if samples of different thickness $d$ are available, then a plot of $N_{T}$ vs d will have an intercept $N_{T}(1 \mathrm{MeV})$ at $d=0$. Such an analysis will allow comparison with DLTS results, which are concerned only with near-surface defects and thus can be analyzed by assuming a constant energy of $1 \mathrm{MeV}$. 


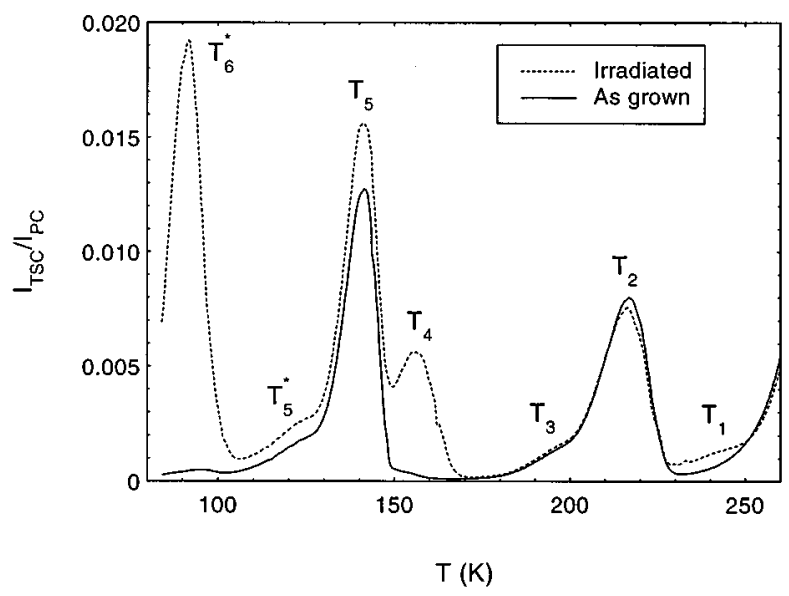

FIG. 1. Normalized thermally stimulated current spectra of asgrown and irradiated $\left(5 \times 10^{14}, 1-\mathrm{MeV}\right.$ electrons $\left./ \mathrm{cm}^{2}\right)$ semiinsulating GaAs.

\section{EXPERIMENTAL RESULTS}

Three adjacent $6 \times 6 \mathrm{~mm}^{2}$ pieces were cut from a 100 $\mathrm{mm}$-diameter, $615-\mu \mathrm{m}$-thick SI GaAs wafer grown by the low-pressure liquid-encapsulated Czochralski method. Halleffect analysis determined a resistivity of $3.4 \times 10^{7} \Omega \mathrm{cm}$, a mobility of $7200 \mathrm{~cm}^{2} / \mathrm{V} \mathrm{s}$, and a carrier concentration of $2.6 \times 10^{7} \mathrm{~cm}^{-3}$, all at $296 \mathrm{~K}$. Transmission measurements [see the discussion following Eq. (5)] gave $N_{E L 2}^{0}=1.5 \times 10^{16}$ $\mathrm{cm}^{-3}$, and $N_{E L 2}^{+}=1 \times 10^{15} \mathrm{~cm}^{-3}$, typical results for such wafers. Ohmic contacts were formed from In dots on the sample corners, and the In was annealed at $425{ }^{\circ} \mathrm{C}$ for 5 min. Current was passed between diagonal contacts. (The geometric factor in such a case is not $\mathrm{wd} / \ell$, but it cancels out anyway in the ratio $I_{\mathrm{TSC}} I_{\mathrm{PC}}$.) One of the three samples was lapped to $415 \mu \mathrm{m}$, and another to $215 \mu \mathrm{m}$, in order to apply Eq. (9).

Electron irradiation was carried out in a van de Graaff accelerator capable of supplying $40 \mu \mathrm{A}$ of $2.2-\mathrm{MeV}$ electrons. However, in this experiment, a $1-\mathrm{MeV}$ beam of electrons was passed through approximately $10 \mathrm{~cm}$ of air before hitting the target. This resulted in a target flux of only 250 $\mathrm{nA} / \mathrm{cm}^{2}$ that was applied for 6 min, giving a total dose of $5 \times 10^{14} 1-\mathrm{MeV}$ electrons $/ \mathrm{cm}^{2}$. This small dose had only a slight effect on the electrical properties, such as the dark current and photocurrent, but was large enough to produce substantial changes in several trap concentrations. NTSC data for the $215-\mu \mathrm{m}$-thick sample are presented in Fig. 1. Clearly, very large increases of traps $T_{6}^{*}$ and $T_{4}$ occur, smaller increases of traps $T_{5}, T_{5}^{*}$, and $T_{1}$, and no measurable increase of traps $T_{2}$ and $T_{3}$. Data for all other samples investigated by us look substantially the same. Quantitative, least-squares fits to Eq. (5), without any approximations, were carried out for traps $T_{6}^{*}(80-110 \mathrm{~K}), T_{5}$, and $T_{4}(130-$ $170 \mathrm{~K})$. The latter two traps had to be fitted simultaneously, i.e., $I_{\mathrm{TSC}} / I_{\mathrm{PC}}=\left(I_{\mathrm{TSC}} / I_{\mathrm{PC}}\right)_{5}+\left(I_{\mathrm{TSC}} / I_{\mathrm{PC}}\right)_{4}$, because of the strong overlap. Excellent fits for all traps were achieved, as shown in Fig. 2 for traps $T_{5}$ and $T_{4}$ of the $215-\mu \mathrm{m}$-thick sample. The fitting parameters are summarized in Table I. The $N_{T}$ value in column 3 is determined by subtracting $N_{T}$ (asgrown) from $N_{T}$ (irradiated) for each of the traps.

From Table I, it is seen that the apparent trap concentrations vary with thickness. This effect was predicted by Eq.

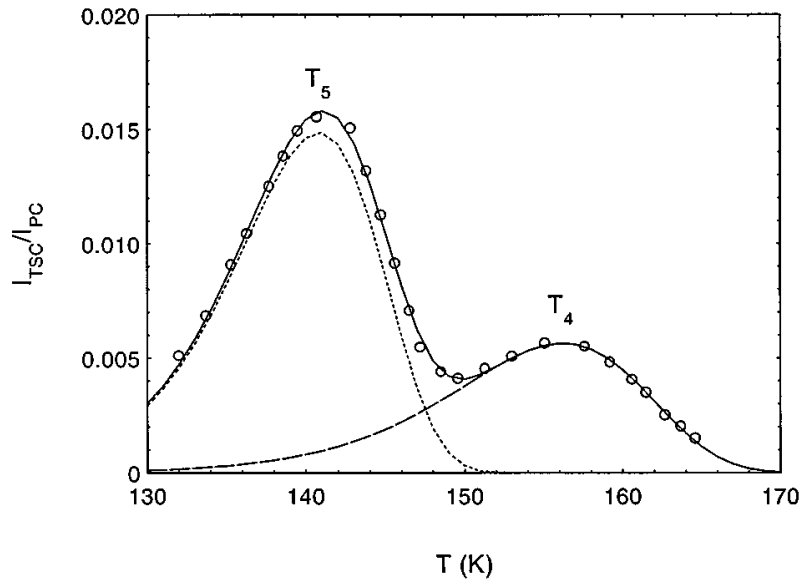

FIG. 2. A fit of the 130-170-K region in Fig. 1, which includes traps $T_{5}$ and $T_{4}$.

(9) and is due to the electron energy loss in the thick samples. Plots of $N_{T}$ vs $d$ for traps $T_{6}^{*}, T_{5}$, and $T_{4}$ are shown in Fig. 3, and straight lines are clearly found for traps $T_{6}^{*}$ and $T_{5}$, although the relationship for $T_{4}$ is more dubious. The concentration values extrapolated to $d=0$ [i.e., $N_{T}(1$ $\mathrm{MeV}$ ) in Eq. (7)] are given in Table I, and lead to production rates of $0.70,0.078$, and $0.23 \mathrm{~cm}^{-1}$ for traps $T_{6}^{*}, T_{5}$, and $T_{4}$, respectively. The corresponding values of $\left[R(E)-R\left(E_{\mathrm{th}}\right)\right]$ are 920,840 , and $1530 \mu \mathrm{m}$, respectively, but the last value should be discarded, because the fit to Eq. (9) for trap $T_{4}$ is not nearly as good as the fits for the other two traps. (With only three points, if one of them is off, the whole fit is compromised.) As mentioned earlier, a numerical calculation of $[R(1 \mathrm{MeV})-R(0.27 \mathrm{MeV})]$, from detailed nuclear scattering theory, ${ }^{12}$ gives a value $970 \mu \mathrm{m}$, in good agreement with our fits of the $T_{6}^{*}$ and $T_{5}$ data. Thus, we evidently have included the electron energy-loss effect properly. It is recommended that such an analysis be employed for all investigations which use GaAs samples of normal thickness $(600-800 \mu \mathrm{m})$ and which involve the whole bulk. As mentioned earlier, however, DLTS analysis is not affected by energy loss be-

TABLE I. Fitting parameters for traps $T_{6}^{*}, T_{5}$, and $T_{4}$ in samples of thickness $(d) 215,415$, and $615 \mu \mathrm{m}$, subjected to irradiation by $5 \times 10^{14} 1 \mathrm{MeV}$ electrons $/ \mathrm{cm}^{2}$.

\begin{tabular}{ccccc}
\hline \hline$d(\mu \mathrm{m})$ & Trap & $N_{T}\left(10^{14} \mathrm{~cm}^{-3}\right)$ & $E_{a}(\mathrm{eV})$ & $\sigma_{a}\left(\mathrm{~cm}^{2}\right)$ \\
\hline \multirow{2}{*}{215} & $T_{6}^{*}$ & 3.10 & 0.13 & $4 \times 10^{-19}$ \\
& $T_{5}$ & 0.34 & 0.34 & $3 \times 10^{-14}$ \\
& $T_{4}$ & 1.10 & 0.31 & $7 \times 10^{-17}$ \\
415 & $T_{6}^{*}$ & 2.72 & 0.12 & $3 \times 10^{-19}$ \\
& $T_{5}$ & 0.29 & 0.35 & $4 \times 10^{-14}$ \\
& $T_{4}$ & 0.95 & 0.32 & $4 \times 10^{-16}$ \\
615 & $T_{6}^{*}$ & 2.33 & 0.12 & $1 \times 10^{-19}$ \\
& $T_{5}$ & 0.25 & 0.33 & $1 \times 10^{-14}$ \\
& $T_{4}$ & 0.94 & 0.30 & $5 \times 10^{-17}$ \\
$0^{\mathrm{a}}$ & $T_{6}^{*}$ & 3.51 & & \\
& $T_{5}$ & 0.39 & & \\
& $T_{4}$ & 1.15 & & \\
& & & & \\
\hline
\end{tabular}

a $y$-axis intercept $(d=0)$ of $N_{T}$ vs $d$ plot. 


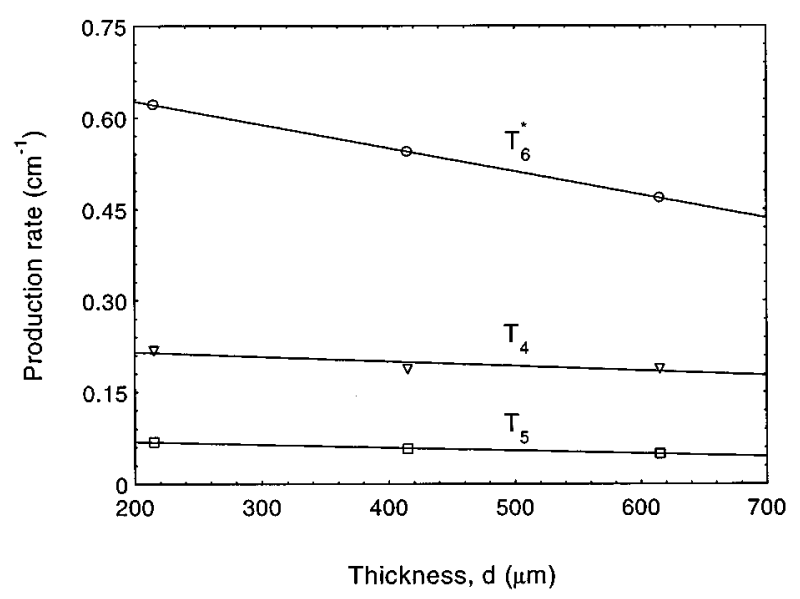

FIG. 3. The 1-MeV-electron production rates of traps $T_{6}^{*}, T_{5}$, and $T_{4}$ as a function of sample thickness.

cause it involves only a small region (typically $0.1-1 \mu \mathrm{m}$ ) near the surface.

\section{DISCUSSION}

The main $1 \mathrm{MeV}$ irradiation traps found by DLTS in conductive, $n$-type GaAs samples are $E 1 \quad\left(E_{a}=0.045 \mathrm{eV}\right.$, $\left.\sigma_{a}=2 \times 10^{-15} \mathrm{~cm}^{2}, r=1.5 \mathrm{~cm}^{-1}\right), E 2\left(0.14 \mathrm{eV}, 1 \times 10^{-13}\right.$ $\left.\mathrm{cm}^{2}, 1.5 \mathrm{~cm}^{-1}\right)$, and $E 3\left(0.30 \mathrm{eV}, 6 \times 10^{-15} \mathrm{~cm}^{2}, 0.4 \mathrm{~cm}^{-1}\right)$, where $r$ is the production rate. ${ }^{3}$ Since the $E 1$ peak would occur well below $80 \mathrm{~K}$ in the TSC experiment (as it does also in the DLTS experiment), we do not see a TSC peak analogous to $E 1$ with our apparatus. However, we see strong similarities between $E 2$ and $T_{6}^{*}\left(0.13 \mathrm{eV}, 4 \times 10^{-19} \mathrm{~cm}^{2}, 0.7\right.$ $\left.\mathrm{cm}^{-1}\right)$, and between $E 3$ and $T_{4}\left(0.31 \mathrm{eV}, 1 \times 10^{-16} \mathrm{~cm}^{2}, 0.2\right.$ $\mathrm{cm}^{-1}$ ). That is, (1) in each case (DLTS and TSC), they are the main irradiation traps; (2) their energies ( $E 2$ with $T_{6}^{*}$, and $E 3$ with $T_{4}$ ) are very close; and (3) their respective production rates are within a factor 2 . Only the capture cross sections differ greatly, especially between $E 2$ and $T_{6}^{*}$.

Regarding the production rates, we notice that the ratios $r(E 2) / r(E 3)$, and $r\left(T_{6}^{*}\right) / r\left(T_{4}\right)$, are equal, within error; thus, it seems that a systematic error could be present in the NTSC analysis, the DLTS analysis, or both. In the NTSC case, the light intensity $I_{0}$ has some degree of uncertainty, because its measurement is accomplished by replacing the whole sample stage with a calibrated photodetector, and it is difficult to ensure that the sample and detector are in the exact same positions. For the DLTS case, calibration is accomplished through measurement of the background shallow donor concentration by the $C-V$ technique, and this method also has sources of error, such as the determination of the diode area $A\left(n_{C V} \sim A^{-2}\right)$. Thus, perhaps a factor 2 between the DLTS and NTSC production rates should not be considered unreasonable. It is also possible, of course, that the primary defect (probably $V_{\mathrm{As}}$ ) production rate in SI material is inherently lower than that in a conductive material, due perhaps to charge-state effects. However, a recent Hall-effect study $^{4}$ of the $0.15-\mathrm{eV}$ defect in conductive GaAs finds a production rate of $0.6 \mathrm{~cm}^{-1}$, very close to our NTSC value. At this point, we do not know why the DLTS production rate differs from that found by the NTSC and Hall-effect methods.

The differences in apparent capture cross section $\sigma_{a}$ are more difficult to resolve; however, several points can be noted in this regard. (1) In the DLTS case, and usually in the TSC case (but not here), cross sections are measured as an intercept of an Arrhenius plot. ${ }^{5,11}$ Because a slight error in slope (or $E_{a}$ ) gives a large error in the intercept $\left(\sigma_{a}\right)$, the latter can be very inaccurate. As an example, in a DLTS study of the same sample by several different laboratories, the $E_{a}$ of $E L 2$ varied only from $0.72-0.84 \mathrm{eV}$, but the $\sigma_{a}$ varied from $4 \times 10^{-17}$ to $5 \times 10^{-15} \mathrm{~cm}^{2}$, while the "accepted" value is $1 \times 10^{-13} \mathrm{~cm}^{2}$ (Ref. 11, p. 201). In our comparison, we are determining $\sigma_{a}$ by two different methods (DLTS and NTSC), and in two different types of GaAs (conducting and semi-insulating, respectively). Thus, large disagreements in the values of $\sigma_{a}$ should perhaps be expected. (2) The DLTS experiment is affected only by the surface region (typically $0.1-1.0 \mu \mathrm{m}$ ), and also involves a very high electric field (typically $10^{4}-10^{5} \mathrm{~V} / \mathrm{cm}$ ). Such high fields can greatly affect emission rates. (3) Note that the $T_{5}$ and $T_{4}$ energies are quite similar, 0.31 and $0.34 \mathrm{eV}$, respectively, and their respective NTSC peaks are close enough that they might not be resolved in the DLTS experiment. Since $\sigma_{a}$ for $T_{5}$, $\sim 3 \times 10^{-14} / \mathrm{cm}^{2}$, is much higher than that of $T_{4}$, the combination of the two $\sigma_{a}$ 's might be similar to that of $E 3$. The same situation might apply to the $E 2 / T_{6}^{*}$ combination; i.e., there could be another NTSC trap, with a similar energy to that of $T_{6}^{*}$, but a higher cross section. Its peak could occur below $83 \mathrm{~K}$, in which case we would not see it. The DLTS defect $E 2$ might then be a combination of $T_{6}^{*}$ and this unseen NTSC peak. However, without lower-temperature measurements we cannot resolve this issue.

\section{SUMMARY}

We have developed a form of thermally stimulated current measurements, called normalized TSC, or NTSC, which eliminates uncertainties due to mobility, lifetime, and geometric factors, but which adds a new factor, the absorption coefficient. Fortunately, the infrared absorption coefficient in semi-insulating GaAs is well known and thus allows the NTSC technique to be completely quantitative in this material. Also, our analysis does not invoke the usual approximations (e.g., an Arrhenius plot of peak positions for different temperature sweep rates), but instead the whole NTSC spectrum, for only one sweep rate, is fitted exactly to the derived formula. This methodology was applied to $1-\mathrm{MeV}$ electron irradiation in semi-insulating GaAs. Three irradiation traps were found: $T_{6}^{*}$ at $0.13 \mathrm{eV}, T_{5}$ at $0.34 \mathrm{eV}$, and $T_{4}$ at $0.31 \mathrm{eV}$. Of these traps, only $T_{5}$ is commonly found at high concentrations in unirradiated SI GaAs; $T_{6}^{*}$ and $T_{4}$ are sometimes found in low concentrations, although they may involve slightly different configurations (complexes) than those produced by the irradiation. The two main traps observed in conductive GaAs, by DLTS, namely, E2 and $E 3$, are identified with $T_{6}^{*}$ and $T_{4}$, respectively. (However, $E 3$ may involve both $T_{4}$ and $T_{5}$.) From extensive previous analysis of the DLTS defects, we then identify $T_{6}^{*}$ with the isolated As vacancy $V_{\mathrm{As}}$, and $T_{4}$ and $T_{5}$ with $V_{\mathrm{As}}-\mathrm{As}_{\mathrm{i}}$ complexes. 


\section{ACKNOWLEDGMENTS}

We wish to thank M. Mier for the absorption measurements, T. Cooper for Hall measurements, L. Callahan for sample preparation, and R. Heil for manuscript preparation.
D.C.L. and Z-Q.F. were supported by U.S. Air Force Contract No. F33615-95-C-1619, and part of the work was performed at the Avionics Directorate, Wright Laboratory, Wright-Patterson Air Force Base, Ohio.
*Permanent address: Physics Department, Khon Kaen University, Khon Kaen, 40002, Thailand.

${ }^{1}$ B. T. Kelly, Irradiation Damage to Solids (Pergamon, London, 1966).

${ }^{2}$ D. V. Lang, in Radiation Effects in Semiconductors, 1976, edited by N. B. Urli and J. W. Corbett, IOP Conf. Proc. No. 31 (Institute of Physics, London, 1977), p. 70.

${ }^{3}$ D. Pons and J. C. Bourgoin, J. Phys. C 18, 3839 (1985).

${ }^{4}$ B. Ziebro, J. W. Hemsky, and D. C. Look, J. Appl. Phys. 72, 3839 (1985).

${ }^{5}$ D. C. Look, in Deep Levels, Gas, Alloys, Photochemistry, edited by R. Willardson and A. Beer, Semiconductors and Semimetals
Vol. 19 (Academic, New York, 1983), p. 75.

${ }^{6}$ M. Tomozane, Y. Nannichi, I. Onodera, T. Fukase, and F. Hasegawa, Jpn. J. Appl. Phys. 27, 260 (1988).

${ }^{7}$ Z-Q. Fang and D. C. Look, Appl. Phys. Lett. 59, 48 (1991).

${ }^{8}$ D. I. Desnica, J. Electron. Mater. 21, 463 (1992).

${ }^{9}$ P. Silverberg, P. Omling, and L. Samuelson, Appl. Phys. Lett. 52, 1689 (1988).

${ }^{10}$ S. K. Brierley and D. S. Lehr, Appl. Phys. Lett. 53, 2426 (1989).

${ }^{11}$ D. C. Look, Electrical Characterization of GaAs Materials and Devices (Wiley, New York, 1989).

${ }^{12}$ W. Brandt, Research Report, Radiation Physics Laboratory, E. I. DuPont and Co., Wilmington, DE, 1960 (unpublished). 